\title{
DETECTAR CONCEPCIONES: EL TEST DE LA NEGACIÓN *
}

\author{
Maribel Narváez Mora \\ Universidad de Girona
}

RESUMEN. En el ámbito de las ciencias sociales se utilizan con frecuencia expresiones con apariencia de enunciados teóricos (externos). Del análisis de las negaciones formales de tales enunciados resulta que su función es menos teórica y más conceptual de lo que aparentan (internos). Suponer la verdad de tales negaciones impide el uso de los términos con el significado que tenían en las afirmaciones originales. Las polémicas en tales casos se caracterizan entonces por ser discusiones entre concepciones y no entre descripciones opuestas de un mismo fenómeno o realidad. ceptos.

Palabras clave: concepción, enunciados descriptivos, relación interna entre con-

ABSTRACT. In the Social Sciences field expressions that appear to be theoretical statements (external) are frequently used. Nevertheless, by analysing their formal negations we realised that their function is less theoretical and more conceptual (internal) than it seems to be. To suppose the truth of such negations preclude the possibility of the involving terms having the same meaning they had in the original affirmative statements. Controversies then are characterized better as discussions between conceptions than as opposed descriptions of the same phenomena or reality.

Keywords: conception, descriptive statements, internal relations among concepts.

* Fecha de recepción: 3 de marzo de 2011. Fecha de aceptación: 3 de abril de 2011. 


\section{PRESENTACIÓN}

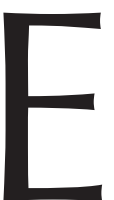

n este texto desearía delimitar y reivindicar un concepto: el de concepción. Delimitarlo para distinguirlo del concepto de teoría, especialmente cuando nos encontramos en el ámbito de las llamadas «ciencias sociales». Aclarar por qué un conjunto de tesis es la expresión de una concepción nos permitirá distinguir teorías que describen y explican fenómenos de concepciones que tienen carácter constitutivo. Reivindicarlo porque creo que es útil detectar las formas de comprensión subyacentes a todo tipo de teorías; es necesario ser conscientes de nuestra metafísica discursiva, así como de que ésta se extiende más allá de lo que estamos en ocasiones dispuestos a admitir. En realidad, hablar de concepciones es algo exagerado, en especial para quien entiende que las concepciones son cosmovisiones o perspectivas vitales de carácter global. No se trata de eso. En el sentido que aquí se va a utilizar, podemos tener una concepción sobre las degustaciones culinarias o sobre los estados de ánimo, los días soleados o los seminarios de teoría del Derecho.

Para hacer manejable la presentación me centraré en ciertas expresiones que se pueden utilizar como premisas o puntos de partida, y a veces como punto de llegada, en todo tipo de confrontaciones argumentativas. Tales expresiones, que se ofrecen a menudo como creencias básicas, y a veces se dice que son las tesis nucleares teóricas de la postura de que se trate, juegan un papel fundamental para la comprensión propia y ajena y no son otra cosa que la expresión parcial de concepciones.

Para mi propósito me fijaré en expresiones generales defendidas con pretensiones teóricas en diversos ámbitos. Como sugería algunas líneas más arriba, centraré la atención en el marco de las ciencias sociales pero, si no voy errada, las implicaciones de lo que sostengo no se ven limitadas a dicho ámbito del saber ${ }^{1}$.

\section{ALGUNOS CONTENIDOS EN LAS LLAMADAS «CIENCIAS SOCIALES»}

En virtud del método de trabajo y de sus objetivos declarados existen ámbitos en ciencias sociales con contenidos marcadamente normativos; otros, por el contrario, tienen una sustancia claramente experimental, empírica y naturalizada. En estos últimos casos las funciones descriptivas y explicativas se pueden satisfacer en mayor o menor medida. Sin embargo, no siempre se hacen explícitos los límites entre funciones descriptivas y el carácter constitutivo de las afirmaciones más generales que cada propuesta utiliza.

1 En filosofía de la ciencia, P. ZEIDLER, por ejemplo, sostiene que los modelos rígidos de estructuras moleculares han sido sustituidos por modelos dinámicos donde la estructura molecular se transforma en virtud de condiciones ambientales. Por ello aunque los modelos teóricos reciban corroboración empírica, y jueguen así un importante papel en la práctica experimental, no pueden entenderse como representaciones de sistemas empíricos reales. De ahí que considere necesario reconocer que se ha producido un cambio en su estatuto epistemológico de los modelos teóricos sobre estructuras moleculares. Vid. P. ZEIDLER, «The Epistemological Status of Theoretical Models of Molecular Structure», International Journal for Philosophy of Chemistry, vol. 6, núm. 1, 2000, 17-34. 
Parte de los fundamentos de tales ciencias son filtrados por un método que podríamos llamar «filosófico». Dicho método está basado en la actividad, perfeccionada por la filosofía analítica del siglo XX, del análisis o reconstrucción conceptual. Desde posturas filosóficas se mantienen también pretensiones descriptivas y explicativas de modo que se sigue diciendo que esta o aquella teoría, acerca de tal o cual cuestión se opone a ésta o aquella otra reclamando corrección o adecuación para sí, en suma, reclamando verdad ya que se supone que desvelan la naturaleza de alguna práctica o fenómeno social.

Podemos pensar en los extremos de un continuo. En uno de ellos se encuentran las propuestas claramente normativas efectuadas y en el otro las aportaciones empíricas de algunos estudios. Una máxima política que defiende ciertos valores o ideología, o un catálogo de pautas éticas que hacen lo propio se situarían con claridad en el extremo prescriptivo. Por su parte, el resultado de ciertos estudios cuantitativos de la sociología empirista o la descripción cualitativa de una práctica pueden situarse en el extremo descriptivo. Existe, sin embargo, un tipo de actividad intelectual que se expresa mediante las correspondientes formulaciones lingüísticas, que no tiene siempre carácter descriptivo-explicativo, a pesar de su forma de presentación y de las explicitaciones metodológicas alegadas. Pero el no tener carácter descriptivo ni explicativo no hace que la actividad en cuestión ni las correspondientes formulaciones, sobre las que vamos a tratar, sean prescriptivas. Es el lugar ocupado por las concepciones. Como he venido diciendo será fundamental la distinción entre lo que es (lo que aquí se entiende por) una tesis teórica y lo que constituye la expresión de una concepción. Por supuesto la semántica de los enunciados involucrados es compleja y no deseo defender «verdaderos significados» fijados de una vez por todas. Lo relevante será qué características o rasgos configuran el sentido de expresiones teóricas y el de expresión de concepciones, aunque luego se las pueda llamar como se desee ${ }^{2}$.

Tener una concepción es tener una visión integral o parcial sobre cualquier cuestión que puede ser articulada lingüísticamente. Se puede tener una concepción sobre la amistad, o sobre la política internacional, pero también sobre los sobrinos y la comida basura. Sería adecuado decir metafóricamente que las concepciones son perspectivas, enfoques o puntos de vista. En tanto que autocomprensiones pueblan el completo espectro de los «saberes». No se elige secundar una concepción antes de tenerla, esto es, no se eligen las reglas de representación con las que dotamos de sentido nuestras acciones verbales y no verbales. No obstante, cuanto más periférica sea la cuestión respecto del conjunto de creencias, con mayor facilidad puede ser abandonada, y con mayor facilidad un enunciado utilizado para expresarla podrá usarse también para efectuar descripciones. Las concepciones pueden ser expresadas lingüísticamente y de las formulaciones que las expresan puede decirse que tienen, en cierto sentido, carácter constitutivo. Por su forma de presentación es fácil malentender su función y considerarlas siempre descripciones.

2 Vale decir que una gran parte de los problemas que quiero tratar proviene del conocido como «giro lingüístico» del Xx. A partir de muchas y muy distintas contribuciones a la filosofía del lenguaje y a la lingüística durante la primera mitad del siglo XX se solidificó un modelo de lenguaje que distingue tres tipos de elementos en la significación: el texto, su significado y aquello a lo que en virtud de dicho significado el texto refiere. Aunque tal partición puede resultar completamente de recibo si se quiere dar cuenta del lenguaje extensional, no parece tener las mismas virtudes cuando lo involucrado son conceptos intensionales. 
«Pero no tengo mi imagen del mundo porque me haya convencido a mí mismo de que sea la correcta; ni tampoco porque esté convencido de su corrección. Por el contrario, se trata del trasfondo que me viene dado y sobre el que distingo entre lo verdadero y lo falso» ${ }^{3}$.

Entre las muchas dificultades que puede enfrentar un ejercicio de delimitación como éste vale la pena mencionar una desde el comienzo. Las concepciones se encuentran, en parte, del lado de las propuestas normativas, en virtud de su naturaleza constitutiva, pero también, en parte, tienen la función de posibilitar descripciones y explicaciones. El hecho de ubicarlas en ese lugar intermedio no significará en ningún caso admitir un híbrido o un punto de equilibrio.

Intentaré argumentar la incapacidad para construir un discurso teórico descriptivo con las formulaciones que expresan concepciones a partir de la prueba de la negación. Con dicha prueba espero que pueda verse que las relaciones que unen los elementos vinculados en enunciados generales expresan concepciones, lo que significa que tienen carácter interno. De ahí que quienes aparecen como contrincantes utilizando tal tipo de expresión no estén participando de una discusión ni sustantiva ni verbal. La discusión no es sustantiva si a este tipo de discusión se le exige la existencia de acuerdo semántico ya que éste no se produce. No obstante, tampoco es meramente verbal, si por ello se entiende una polémica que puede disolverse sólo con poner de manifiesto que quienes discuten dan significados distintos a los términos que utilizan. Vale decir que las expresiones por sí mismas no enuncian concepciones, ello dependerá de las funciones que estén cumpliendo. En algunos casos no es posible aislar un mínimo universal que caracterice a los fenómenos para los que utilizamos conceptos intensionales, para luego predicar de éste tal o cual característica, especialmente en los casos de máxima generalidad: llegado cierto nivel no se dispone de concepciones alternativas en un marco de referencia dado ${ }^{4}$.

\section{MI PRIMER USO DEL TEST DE LA NEGACIÓN}

Hace algún tiempo decidí someter a prueba las afirmaciones de los políticos durante las campañas electorales. El objetivo era establecer con alguna claridad qué contenido sustantivo se defendía al hacer tales afirmaciones para poder emitir un voto lo más informado posible ${ }^{5}$, aunque las más de las veces de lo que se trataba era de enten-

3 L. WitTgenstein, Sobre la certeza, Barcelona, Gedisa, 1995, $\$ 94,15$ c.

${ }^{4}$ Como espero que pueda verse el término del texto con el ejemplo del rey Darío, al hablar de marco de referencia aludo al punto de mínimo común denominador que se requiere para sostener de dos tipos de algo que lo son de ese algo. En el ejemplo que ofreceré más adelante el rey Darío dispone de ese marco. Otro ejemplo simple podría ser el siguiente: en la comunidad A los conocidos se saludan con un apretón de manos, y en la comunidad B se saludan llevando la palma de la mano derecha extendida hasta el lado izquierdo del pecho. Contamos con una noción de saludo (marco de referencia) que nos consiente decir que estamos en presencia de dos tipos de saludo. Tanto es así que si en la comunidad C, por hipótesis, los conocidos se saludasen dándose patadas con fuerza al verse, no podríamos identificar el supuesto como el de un saludo. Rechazaríamos que ese es otro tipo de saludo. Cuando redacto estas líneas me convierto en el rey Darío generando la hipótesis de que ése es el saludo de la comunidad C. Ésa es una ficción narrativa.

5 Uno de los valores superiores del ordenamiento jurídico, según consagra la Constitución española de 1978, es el pluralismo político. Dicho valor parece salvaguardarse cuando existe una «opinión pública bien formada». La idea de que los medios tienen especial relevancia en la materialización de dicha formación se di- 
der qué decían los programas y en qué se distinguían unos de otros. La sospecha que me llevó a poner en práctica la «prueba» fue que la grandilocuencia de las expresiones no parecía tener nada que ver con la diminuta relevancia del contenido expresado. Denominé a dicha prueba «el test de la negación» y más adelante lo definiré para el contexto que nos interesa de la teoría del Derecho.

Pero ahora me mantendré en este contexto de descubrimiento ¿En qué consistía el test por lo que hacía a las campañas electorales? Tomaba cualquiera de los enunciados que encontraba en un programa electoral, o cualquiera de las manifestaciones de los líderes políticos durante la campaña, y construía su negación formal. A continuación me formulaba la pregunta: ¿Habrá alguien dispuesto a defender la negación en cuestión, algún partidario de lo contrario de lo que aquí se dice? Ya sabemos que hay gente para todo, pero si la respuesta que yo daba a la pregunta era «No, no creo que lo haya, al menos entre los llamados a votar en estas elecciones», entonces me parecía claro que la afirmación en cuestión carecía de contenido político. Por el contrario, cuando la respuesta era sí, la afirmación de origen tenía un contenido político que estaba en juego al decantarme por una u otra formación política. Tener contenido, políticamente hablando, supone bajo el prisma de este test, que lo dicho es objeto de debate y que resulta relevante para «tomar partido», que es de lo que se trata al elegir representantes.

Con la ayuda de la prueba de la negación podemos analizar enunciados de tipo diverso en el discurso político. Por ejemplo, compromisos electorales. Si se lee «Trabajaremos para superar los estereotipos de género» ${ }^{6}$, se observa que tanto la negación externa de la expresión: «No trabajaremos para superar los estereotipos de género», como la interna: «Trabajaremos para no superar los estereotipos de género», no pueden ser secundadas, al menos en los escenarios electorales a los que me estoy refirien$\mathrm{do}^{7}$. Por su parte, si se dice: «Queremos introducir una tasa sobre las transacciones financieras internacionales» ${ }^{8}$ puede construirse con más facilidad un debate en torno a dicha propuesta y su negación. Algunos (muchos o pocos) — de ahí la idea de opinión mayoritaria o minoritaria- estarán a favor y algunos (muchos o pocos) en contra de la creación de dicha tasa.

Para comunicar tales compromisos electorales hace falta articular una serie de creencias y deseos con los que se construyen las reglas técnicas que sirven de base a las distintas políticas.

Las articulaciones de deseos no pasan el test de la negación cuando son en realidad la expresión de lo que consideramos un deseo generalizado o universal. Sien-

funde a partir de pronunciamientos judiciales del más alto nivel (Tribunal Europeo de Derechos Humanos, 23 de abril de 1992, STC 190/1992, de 16 de noviembre). Por su parte, la idea de que la responsabilidad de estar bien informado es un mandato para el ciudadano republicano, podemos encontrarla en muchos lugares.

${ }_{6}^{6}$ Propuesta en el programa electoral para las elecciones autonómicas 2010 del Partido Socialista de Cataluña en http://www.presidentmontilla.cat/files/programaelectoral-psc-2010.pdf.

7 Con independencia de que alguien verdaderamente desee que las cosas sean así, la regla del discurso público impone unas restricciones, al menos temporalmente. Nada excluye un concreto contexto socio-político en el que se escuche a un candidato sostener: «Trabajaremos para no superar los estereotipos de género». De hecho, no creer en las promesas electorales sólo significa muchas veces que creemos que el resultado final de las políticas se parecerá a lo contrario de lo dicho.

8 Propuesta en el programa electoral para las elecciones autonómicas 2010 del Partido Socialista de Cataluña en http://www.presidentmontilla.cat/files/programaelectoral-psc-2010.pdf. 
do algo esquemáticos podría decirse que puesto que no hay quien no desee eso, afirmar que es eso lo que se desea conseguir tiene garantizado el apoyo. «Apostamos por un modelo político que permita a los ciudadanos vivir con más libertad y desarrollar sus propias potencialidades» ${ }^{9}$. Por su parte, la articulación de creencias, las afirmaciones descriptivas sobre hechos, no pasan la prueba cuando resultan obviedades; por el contrario, superan el test al no ser creencias compartidas por todos. «Creemos que la Administración ha invadido demasiados espacios de la sociedad $\gg^{10}$.

En otras ocasiones el discurso electoral se centra en vaticinios de futuro para criticar o avalar ciertas medidas. Se toma entonces un referente independiente, por ejemplo «La nueva ley $\mathrm{X}$ », y se sostiene que su puesta en vigor llevará a (producirá, causará, tendrá las consecuencias) Y. Si el partido en la oposición dice que la puesta en vigor de X llevará a Y, el partido en el gobierno suele decir justo lo contrario. Al auditorio, en general a la ciudadanía, se le lleva así a la encerrona de la inferencia. Si apoyas la puesta en vigor de $\mathrm{X}$ apoyas que se produzca la consecuencia $\mathrm{Y}$. Puesto que no hay quien desee $\mathrm{Y}$, y a ese absurdo te llevaría defender la nueva ley X. Tú no puedes desear realmente eso, ¿verdad?

Aquí la controversia (pero sólo de la clase política) está garantizada. Para el militante de un partido decir «ley $\mathrm{X}$ », se vincula discursivamente a los efectos perniciosos sobre los que tanto nos han advertido. Decir «ley X», para el militante del partido rival está asociado a unos efectos beneficiosos incuestionables.

Aparentemente los enunciados de futuro tienen cierta ventaja, frente a los que mencionábamos al inicio. Puesto que algo efectivamente ocurrirá tras la entrada en vigor de $\mathrm{X}$, tal vez podamos evaluar los efectos de $\mathrm{X}$ y comprobar quien tenía razón. Pero la apariencia queda en eso. Prácticamente sean cuales sean los efectos, se magnifican, por parte de los interesados, los beneficios o desventajas obtenidos dependiendo de la posición política de origen. Con esta vuelta de tuerca se inicia el camino donde lo habíamos dejado.

MERTON explicó hace ya largo tiempo cómo, en ciertas condiciones, se auto-cumplen las profecías en la vida social. Resulta asombroso que nadie se avergüence de construir un determinado tipo de discurso político, por cierto, muy generalizado. Aquí no voy a hablar de comportamiento electoral ni haré un análisis del discurso político. No hace falta dedicarse a ninguna actividad académica para darse cuenta de los devastadores efectos de tales prácticas en la vida pública. De la vacuidad evidente, con o sin test de la negación, se pasa al absentismo electoral, de ahí al rechazo activo ${ }^{11}$, y la clase política parece no saber que «hay un mundo ahí afuera».

9 En el programa electoral para las elecciones autonómicas 2010 del Partido Popular de Cataluña en http://www.ppcatalunya.com/wp-content/uploads/2010/programa_castellano_LWR1.pdf.

${ }^{10}$ En el programa electoral para las elecciones autonómicas 2010 del Partido Popular de Cataluña en http://www.ppcatalunya.com/wp-content/uploads/2010/programa castellano LWR1.pdf.

${ }^{11}$ El Centro de Investigaciones Sociológicas recoge en su estudio núm. 2.856 del mes de diciembre de 2010 el resultado que vemos en porcentajes como respuesta a la pregunta 18, formulada del siguiente modo (repárese en la tercera de las líneas «la política»): «A lo largo de la vida para una persona hay cosas que son muy importantes, mientras que otras, por el contrario, carecen de importancia. Para cada una de las cuestiones que voy a leer a continuación, ¿podría decirme si representan para Ud. en su vida, algo muy importante, bastante, poco o nada importante?». 
En conclusión: la expresión de deseos y creencias ampliamente compartidos son una garantía, suponen poco riesgo porque carecen de contenido político. La cuestión de fondo es sencilla: digamos cosas que nadie pueda negar, ya que negarlas o admitir su negación sería absurdo, carece de sentido.

\section{EN OTRO ORDEN DE COSAS, EL QUE NOS INTERESA}

Pero fijarme en el discurso electoral y en lo que a éste le ocurre cuando se le aplica el test de la negación es sólo algo instrumental. Lo que sí me interesa es la idea de fondo con la que cerraba el apartado anterior: que existan expresiones cuya negación desde la perspectiva de quienes las formulan, carezca de sentido.

En el ámbito de la teoría del Derecho no hay afirmación que formule una teoría que no sea puesta en cuestión por otra, así que negar lo que otro sostiene está al orden del día. Dicha discusión está cimentada, precisamente, en cuestionar las tesis centrales de los oponentes. Podría decirse que, contrariamente a lo que ocurre en el discurso político, en el debate iusteórico no se verifica el refrán «a río revuelto ganancia de pescadores», razón por la cual delimitar claramente la propia posición es un requisito.

El test de la negación ¿nos puede ayudar a tomar partido en el debate iusteórico? El test no nos brindará la ayuda necesaria para destilar opciones correctas. Lo que nos va a permitir es detectar polémicas construidas sobre no distinguir reglas de representación del resultado de haber usado dichas reglas. Usando el símil para las longitudes, sobre no distinguir el metro en tanto que patrón de medición y el metro como resultado de la medición. Una cosa es expresar una concepción sobre el Derecho y otra describir o evaluar fenómenos en el marco de dicha concepción.

En primer lugar será necesario presentar algunos conceptos básicos. También, por supuesto, necesitamos decir algo acerca de la noción de teoría a la que me refiero ${ }^{12}$.

En segundo lugar utilizaremos algunos debates al uso para ejemplificar lo dicho. La conclusión del análisis será poco halagüeña si lo que se pretende es dar con expre-

\begin{tabular}{|l|r|r|r|r|r|r|c|}
\hline \hline & Muy & Bastante & Algo & Poco & Nada & N.S. & N.C. \\
\hline El trabajo & 62,1 & 30,0 & 1,9 & 2,1 & 2,8 & 0,4 & 0,7 \\
\hline La familia & 86,0 & 12,7 & 0,8 & 0,2 & 0,2 & 0,0 & 0,1 \\
\hline La política & 6,1 & 21,3 & 16,4 & 31,7 & 23,4 & 0,6 & 0,5 \\
\hline El bienestar económico & 44,0 & 50,9 & 3,7 & 0,8 & 0,0 & 0,1 & 0,5 \\
\hline El tiempo libre/ocio & 35,2 & 50,9 & 8,3 & 4,2 & 0,6 & 0,2 & 0,6 \\
\hline La salud & 86,9 & 12,4 & 0,4 & 0,1 & - & 0,0 & 0,2 \\
\hline Los/as amigos/as & 41,9 & 48,0 & 7,0 & 2,1 & 0,3 & 0,4 & 0,2 \\
\hline La religión & 8,4 & 20,2 & 16,5 & 29,9 & 24,2 & 0,5 & 0,2 \\
\hline Las relaciones de pareja & 54,0 & 36,0 & 3,4 & 3,1 & 2,0 & 0,7 & 0,9 \\
\hline
\end{tabular}

12 «I will be using "a theory of law" in a narrow sense, as referring to an explanation of the nature of law. [...] The thesis I will de defending is that a theory of law is successful if it meets two criteria: first, it consists of propositions about the law which are necessarily true, and second, they explain what the law is». J. RAZ, Can be a Theory of Law? The Balckwell Guide to the Philosophy of Law and Legal Theory, M. P. Golding y W. A. Edmundson, Oxford, Blackwell, 2005, 324-342, esp. 324. 
siones aptas para la verdad, que podría ser el mínimo exigible para el contenido de las tesis de cualquier teoría.

\section{PROPOSICIÓN. AFIRMACIÓN Y NEGACIÓN}

Son muchas las definiciones de «proposición» que se manejan en el ámbito filosófico. Éstas guardan relación en algunos supuestos con lo que enseñan la lingüística, la semántica y por supuesto la lógica, aunque no siempre se puede obtener una visión panorámica clara. Podemos decir que la proposición es el contenido, o significado, de una oración que puede ser verdadera o falsa, pero no ambas cosas a la vez. También se sostiene que una proposición es el contenido de una oración, es decir, el conjunto de situaciones (a veces mundos posibles) en las cuales la oración es verdadera.

Una frase, como conjunto de grafías o palabras, no es una proposición. La frase u oración, se nos dice, es la forma lingüística en un idioma determinado que puede expresar una proposición.

Otra manera de definir «proposición» es considerarla la unidad semántica de la cual puede predicarse verdad o falsedad. Las órdenes o recomendaciones no pueden ser verdaderas o falsas: de ellas decimos más bien que son juiciosas o insensatas, seguidas o ignoradas, eficaces, justas o válidas. Verdad es la propiedad de la unidad semántica que describe adecuadamente, correctamente, el mundo. Verdad y falsedad pueden considerarse también simplemente como los valores lógicos de la unidad semántica descriptiva con sentido completo.

Pues bien, tomando cualquiera de estos puntos de partida cabe decir que si una frase, oración o enunciado (de ahora en adelante enunciado) expresa una proposición, también la negación de dicho enunciado expresa una proposición.

El punto crucial se encuentra en la distinción entre negación formal (no interpretada) y negación sustantiva o interpretada.

Veamos un ejemplo simple:

E1: El hombre es bueno por naturaleza.

De E1 puede decirse que expresa una proposición siempre que se esté de acuerdo en sostener que lo que expresa es verdadero o falso pero no las dos cosas a la vez y que ése sea el criterio para el uso de «proposición» que se utilice. Por supuesto, apelando a la ambigüedad puede sostenerse que no expresa proposición alguna argumentando, por ejemplo, que es una manifestación encubierta o implícita de deseos. Obviamente, si se rechaza la definición de «proposición» asumiendo que algunas proposiciones tienen valores de verdad distintos a verdadero y falso, mantener que E1 expresa una proposición no nos comprometería con el tercero excluido, que en general sí vamos a necesitar para nuestro propósito.

Mantengamos por el momento la postura más simple: E1 expresa una proposición por lo que o es verdadero o es falso. Bajo esa perspectiva sabemos alguna cosa más. Sabemos que si E1 es verdadero su negación es falsa, y viceversa.

E2: El hombre no es bueno por naturaleza. 
Supongamos que alguien defiende la verdad de E2. Cuando lo hace puede ocurrir que los términos con los que se ha construido la expresión tengan un sentido que no pueda ser el que tenían en E1. Ello ocurre si no hay un referente para «hombre» común en ambos casos del que luego se diga que tiene o no tiene una propiedad, «bondad natural». Si quienes usan el término «hombre», en cada uno de los dos supuestos, lo está dotando de sentido incorporando o excluyendo la «bondad natural» según el caso, no se está realizando la predicación de propiedades opuestas al mismo referente. La relación entre los aparentes sujeto y predicado, por decirlo así, sería interna y, por tanto, no estaríamos ante una auténtica predicación. Si un enunciado es la negación de otro, en el debate, ambos contendientes deben otorgar el mismo sentido a los términos que funcionan como sujeto y predicado. Para la predicación entre particulares o descripciones de objetos sometidos a ubicación espacial y temporal el modelo es de lo más adecuado. El problema es que no siempre estamos materialmente ante una predicación copulativa. Podríamos decir que E1 y E2 no refieren a hombres, sino que nos dicen algo sobre el concepto de hombre, que son parcialmente constitutivos del sentido del término que en ellos se utiliza.

¿Pero eso no significa tan sólo que se están tomando las expresiones E1 y E2 como distintas definiciones parciales de «hombre» o como expresiones analíticas y no sintéticas?

\section{RELACIÓN INTERNA}

Existe un modo erróneo de entender la relación interna, puesto que la presencia de estas relaciones impide la construcción de una negación sustantiva de la expresión. Tomemos una definición material o una expresión analítica, de las que se denominan «verdaderas por definición»: E3: «Todos los solteros son hombres no casados» y sus negaciones formales, E4: «Algunos solteros no son hombres no casados», E5: «Algunos solteros son hombres casados». La idea de relación interna no es meramente la que llevaría al aguijón semántico. Esto es, si E4 y E5 son verdaderos, entonces simplemente se está hablando de algo distinto a lo que se hablaba en E3. Conformarse con la conclusión se «habla de otra cosa» es un error, porque aunque se trata de eso, no sólo se trata de eso.

En el diccionario sobre el trabajo de WiTTGENSTEIN elaborado por H. JOHANN GLOCK ${ }^{13}$, qué cuenta cómo relaciones internas queda definido del modo siguiente: «relations which could not fail to obtain, since they are given with or (partly) constitutive of the terms (objects or relata), such as white's being lighter than black». Esta idea de WitTGENSTEIN proviene del Tractatus, en el que ofrece una definición de propiedad interna:

«Una propiedad es interna si resulta impensable que su objeto no la posea. / (Este color azul y aquel están eo ipso en la relación interna de más claro y más oscuro. Es impensable que estos dos objetos no estuvieran en esa relación)» ${ }^{14}$.

${ }^{13}$ H.-J. GLOCK, A Wittgenstein Dictionary, Oxford, Blackwell, 1996, 189.

${ }^{14}$ L. Wittgenstein, Tractatus Logico-Philosophicus, trad. de J. Muñoz e I. Reguera, Madrid, Alianza, 1987, 3. .a reimpresión, $\$ 4.123,1922,69$. 
Con todo, a lo largo de la evolución del pensamiento de WiTTGENSTEIN, dicha relación dejó de ser entendida tan sólo como propia del lenguaje fenomenológico, y se extendió al lenguaje intencional, sirviendo para entender ideas tan distintas como el seguimiento de reglas o la comprensión de acciones intencionales.

Solicitar una justificación de la relación interna, o plantearse una duda acerca de si dicha relación es predicable de un par de elementos carece de sentido. En cierto modo, la relación es constitutiva de lo relacionado de manera que no es posible, coherentemente, negar la relación sin que se esté «hablando de otra cosa» o baciendo referencia a otro elemento. Pero entenderlo así, como dije, es lo propio de aceptar la corrección del argumento del aguijón semántico ${ }^{15}$.

Si se presupone la verdad de la negación de un enunciado que expresa una concepción, entonces los términos que aparecen en dicha negación no pueden tener el mismo significado que tenían en la concepción de partida. Veamos algunos ejemplos:

a) Supongamos el enunciado «Ningún objeto es simultáneamente completamente rojo y completamente azul», al considerar las condiciones que harían verdadera su negación «Algún objeto es simultáneamente completamente rojo y completamente azul», resulta imposible mantener el significado de todas las palabras en la afirmación y en la negación. Es decir, si la negación fuese verdadera, «completamente», «simultáneamente», «objeto», «rojo» etc., no podrían tener el significado que tienen en la afirmación.

b) Supongamos la verdad de la negación de «No puede existir un mundo en el que todo sea distinto», es decir, la verdad de «Puede existir un mundo en el que todo sea distinto», entonces, «mundo» «todo» o «distinto» no podrían tener el mismo significado en ambas expresiones.

c) Supongamos la verdad de «Puedo sentir el dolor de otros» como la negación de «No puedo sentir el dolor de otros», tenemos que reconocer que en cualquier mundo que el primer enunciado fuese verdadero, «dolor» no podría significar lo mismo que en el mundo en que el segundo enunciado fuese verdadero ${ }^{16}$.

El corolario de estos ejemplos es que mientras los términos tienen el sentido que se les otorga por una concepción su negación carece de sentido. La negación de un enunciado básico en una concepción, que lo está usando como tal, carece de sentido en el sistema de esa concepción ya que éste constituye una regla de representación, y por tanto la expresión que muestra cuál es el sentido de otras ${ }^{17}$.

${ }_{15}$ R. DworkIn, Law's Empire, Cambridge, HUP, 1986.

${ }_{16}$ Que un enunciado sea la negación sustantiva de otro presupone una identidad semántica de algún tipo entre los componentes de los enunciados. Quien admitiese un concepto de proposición de carácter veritativo funcional, tendría que decantarse por, o bien admitir que en ciertos casos las condiciones de verdad de un enunciado no son las condiciones de falsedad de su negación, o bien admitir que si ese tipo de expresiones existe, entonces no expresa una proposición.

${ }_{17}$ Así, Moore escribe en las anotaciones de las clases impartidas por WitTGENSTEIN en Cambridge: «¿Acaso "No puedo sentir su dolor de muelas" no significa que "Siento su dolor de muelas" no tiene sentido?» [Klagge y Nordmann, 1993, 91. La publicación Philosophical Occassions 1912-1951 a cargo de KLAGge y NORDMANN en 1993 recoge con algunas correcciones del propio MoORE los apuntes y comentarios de clase aparecidos en Mind, 1954, 63 (1-15) y (289-315), y 1955, 64 (1-27 y 264) con el título «Las clases de Wittgenstein durante el periodo 1930-1933». La cita se corresponde con una explicación ofrecida por WITTGENSTEIN, según MoORE, sobre el sinsentido de una expresión que mencionó al final del periodo (entre mayo de 1932 y el último trimestre de 1933)]. 
No suele prestarse atención a la formación de las concepciones individuales o colectivas ni a los modos de comprensión subyacentes que otorgan sentido identitario a los sujetos y a las colectividades. Por ejemplo, la concepción de aprendizaje académico que yo poseo, y sobre la que puedo no haber reflexionado jamás, no puede despegarse, desprenderse o disociarse de la idea de creatividad. Ésta puede ser mayor o menor, pero presupongo que algún grado de creatividad está siempre involucrado en el aprendizaje. Podría decir que me resulta inconcebible, que sin creatividad se pueda aprender no porque no pueda imaginar otra concepción de aprendizaje, sino porque nada de lo que digo y hago dotando de sentido por mi propia concepción podría tener el sentido que tiene si el aprendizaje fuese algo meramente mimético o repetitivo: no contaría como aprendizaje académico y no identificaría como tal dicha actividad.

\section{LA POSTURA DE RAZ}

J. RAZ se ha preguntado acerca de la posibilidad de que exista una teoría del Derecho ${ }^{18}$. Para entender el concepto de teoría del Derecho que utiliza valgan sus propias palabras: "I will be using "a theory of law" in a narrow sense, as referring to an explanation of the nature of law. [...] The thesis I will de defending is that a theory of law is successful if it meets two criteria: first, it consists of propositions about the law which are necessarily true, and second, they explain what the law is».

El texto de RAZ es muy estimulante. Señala con claridad algunos de los problemas epistemológicos involucrados en el tratamiento de nociones como significado, concepto y explicación que hasta la fecha se encuentran latentes en el debate iusteórico. RAZ presenta un mapa utilizando los elementos: significado de las palabras, conceptos y sus casos de aplicación.

El modelo teórico constituido por los elementos palabra-significado-cosa es especialmente embaucador. Generalmente se construye y se presenta utilizando ejemplos de la función nominativa del lenguaje, y además para palabras de clase que con frecuencia nombran algún tipo de entidad u objeto empírico. Casi con toda seguridad su aprendizaje escolar trae origen en el proyecto saussuriano de la significación, y su aprendizaje filosófico en las sucesivas construcciones de FREGE, RUSSELL, CARNAP. En este modelo por un lado tenemos palabras, entendidas como símbolos, por otro sus significados, y por último aquello a lo que se refieren. La relación entre el significante o símbolo y el significado es arbitraria, por lo que el uso correcto de la palabra dependerá de cuál sea el significado asociado a ésta. A veces se habla de criterios de uso, y a veces del concepto involucrado.

RAZ entiende que este modelo puede dificultar entender en qué consiste una teoría del Derecho. La forma en que se conciben objetos y fenómenos depende de los conceptos, tanto para el filósofo como para cualquier persona. Los conceptos, nos dice, se encuentran entre los objetos y las palabras. Nos advierte de que si se exagera la relación de los conceptos con las palabras, éstos se llegan a considerar el significado de los tér-

18 J. RAZ, «Can There Be a Theory of Law?», en M. P. Golding y W. A. EDmundson (eds.), The Backwell Guide to the Philosophy of Law and Legal Theory, Oxford, Blackwell, 2005, 324-342. 
minos. Por otro lado, si se hace hincapié en el polo contrario, hablar de conceptos se convierte en hacerlo de la naturaleza de los objetos. En este caso explicar los conceptos y la naturaleza de esas cosas u objetos se convertiría en actividades equivalentes. Hay quien entiende que no hay dificultad en conjugar ambas posturas mediante el análisis conceptual.

Para RAZ, en ambas posturas hay algo correcto, pero también algo erróneo. Los conceptos, nos dice, son cómo concebimos los aspectos del mundo y están entre éste y las palabras y sus significados por una parte y entre las cosas o fenómenos por otra. De esta forma considera, por ejemplo, que HART en su trabajo no estaba diciéndonos nada sobre la palabra «Derecho» dada su forma de excluir muchos de los significados de esa palabra. Por ejemplo, todos los significados relacionados con las normas religiosas y matemáticas quedaron excluidos de su concepto de Derecho. La pretensión es teórica cuando se quiere decir algo sobre la naturaleza de los fenómenos, y ése entiende que era el caso de HART.

RAZ explica su visión a partir de las ideas de poseer y entender un concepto ¿En qué condiciones es verdad que alguien posee y entiende un concepto? Los conceptos difieren entre sí por la información que se necesita para tener y entender uno de ellos, y por las competencias y habilidades involucradas en su posesión. Si entender un concepto es conocer la cosa o fenómeno, comprender un concepto es conocer esa cosa o ese fenómeno conociendo sus rasgos necesarios.

Se tiene competencia completa sobre un concepto si se conocen y comprenden todos los rasgos necesarios de los objetos a los que se aplica. Triángulo equilátero y triángulo equiángulo no son conceptos idénticos aunque las propiedades de estos triángulos sean todas ellas las mismas. Se aplican a las mismas figuras por razones diferentes, en un caso por su propiedad de ser equiláteros y en el otro por ser equiángulos. Así es posible decir que las condiciones mínimas para poseer ambos conceptos son diferentes, y de ahí que sean conceptos distintos. Un completo conocimiento de ambos conceptos haría que se pudiesen utilizar para comprender el tipo de triángulo al que ambas se refieren al saber que tienen las mismas propiedades necesarias y por ello son los mismos triángulos.

RAZ sostiene que las condiciones que una teoría del Derecho debe cumplir para ser tal es que esté integrada por expresiones necesariamente verdaderas del Derecho y que a su vez expliquen el fenómeno. Por ello le resulta útil la propuesta que PuTNAm hizo para abandonar la idea de que el significado de los términos nos da la información necesaria para identificar los fenómenos a los que éstas se refieren. Pero el uso del modelo de Putnam no refuerza la postura de RAz.

Si un concepto es de naturaleza intensional y por ello dependiente de creencias, no cumple con los requisitos que van a permitir el error generalizado, que juega un rol fundamental en el supuesto de las clases naturales. Por eso la comunidad que no tiene nuestro concepto de Derecho no está cometiendo un error generalizado cuando sostiene que es necesariamente verdad del Derecho algo que nosotros consideramos contingente.

Si se dijese que existen varios conceptos de Derecho, entendidos como varios significados para la palabra «Derecho», esto no puede satisfacer la pretensión de RAZ 
ya que la teoría del Derecho no sería un conjunto de proposiciones necesariamente verdaderas del objeto al que le es aplicable nuestro concepto de Derecho.

¿Pero qué ocurre si seguimos a RAZ a partir de nuestra idea de expresión de una concepción? Si se toma una de esas proposiciones, siendo como es necesariamente verdadera, su negación tendrá que ser necesariamente falsa. Para que lo sea, tendrá que ser la negación, no meramente formal, sino sustantiva de la proposición.

Supongamos que una de las proposiciones relevantes en la teoría fuese E6: «El contenido del Derecho no depende de su mérito moral». Si lo dicho en E6 es necesariamente verdadero del Derecho, entonces E7: «El contenido del Derecho depende de su mérito moral» tiene que ser necesariamente falsa, PERO ANTES tiene que ser la negación de la primera. Para que una proposición sea la negación de otra, como dije, es de suponer que los símbolos que se usen representan en ambos casos los mismos conceptos, así que «contenido del Derecho» «depender» «mérito moral», o cualquier otra palabra (de otro lenguaje natural) o símbolo debe mantener esta característica. Ello es necesario si se defiende el principio de composición en el lenguaje. Si no se da esta condición podría ocurrir que ambas expresiones fuesen verdaderas, lo que supondría admitir que una no es la negación de otra. Quien sostiene, en lo que se supone que es un debate entre teorías del Derecho (o entre propuestas que aspiran a llegar a ser teorías del Derecho) que «el contenido del Derecho depende de su mérito moral» es imposible que esté usando el mismo concepto de contenido del Derecho que quien sostiene lo contrario, puesto que en su uso decir «contenido del Derecho» es decir «contenido con mérito moral».

Si una forma de entender que cierta proposición es necesariamente verdadera consiste en decir que establece una relación interna (o un vínculo conceptual) entre los elementos relacionados, entonces negar la relación interna no va a tener éxito —en el sentido de producir una negación del primero-: se convierte para quien parte de ese lugar en un sinsentido. Recuérdese que los enunciados no son objetos que significan, sino que significan para sujetos situados en algún espacio de significación.

Cuando RAZ habla de «proposiciones necesariamente verdaderas» sobre el Derecho, está hablando de lo que no puede ser negado con sentido. Pero dos elementos diferencian su punto de partida con el que aquí se defiende: en primer lugar la idea de verdad no puede aquí jugar un papel, a menos que lo jugase la idea de verdad por definición que es completamente distinta a la idea de verdad por correspondencia o por adecuación de cualquier tipo. Si la expresión es verdadera, su negación es falsa, pero dicha negación en el sistema de reglas de representación de RAZ sería un sinsentido ${ }^{19}$. El segundo punto de diferencia es el carácter de necesidad. RAZ está obligado a echar mano de la noción de mundo posible, de necesidad metafísica o alguna similar para poder hablar de necesidad, no puede llegar a defender la universalidad que requiere su propuesta porque la visión o concepción alternativa queda como una falsedad. Para decirlo de otra manera, si RAZ consigue que los enunciados sean necesariamente verdaderos, no serán explicativos; y si son explicativos, no serán necesariamente verdaderos ${ }^{20}$.

19 Hay que distinguir senseless y meaningless en WITTGENSTEIN, a partir de las comparaciones de su primera y segunda época. Para ello son muy útiles las aclaraciones que hiciera (lectura de videoconferencia) vON WRIGHT en el congreso Wittgenstein research revisited, Bergen (Noruega), 12-15 de diciembre de 2001.

20 Vid. para la discusión de la propuesta de RAZ, J. RAZ, R. AlEXY y E. BulygIN, Una discusión sobre la teoría del Derecho, Madrid-Barcelona, Marcial Pons, 2007. 
El rasgo que puede despertar mayor recelo si seguimos mi línea argumental, y desde luego con razón, es el de la aparente defensa de un relativismo fuerte. Produce pánico decir que si la expresión de partida es «Las mujeres no son seres inferiores a los hombres», aquí lo que tenemos es meramente la expresión de una concepción y que dicho enunciado no expresa una verdad, necesaria o tan sólida como podamos hacerla ser. Si la negación formal «Las mujeres son seres inferiores a los hombres» es a su vez otra expresión de una concepción tenemos el siguiente panorama: quien enuncia la primera tiene que decir que la otra carece de sentido, pero no que es falsa.

La expresión en cuestión tiene, al menos, tres tratamientos posibles.

El primero es el que haría una lectura externa de la relación que establece entre sus elementos. Eso presupone un criterio, por ejemplo biológico, para la clasificación de elementos, y una comparación con criterios a su vez compartidos. Esto es, la primera lectura permite decir que una de ellas es verdadera y la otra falsa. Aquí la lectura tradicional sabe que el criterio biológico podría llegar a decir que se da dicha inferioridad (al menos que hay diferencia biológica), y por eso cabría la segunda lectura: decir que se trata de ofrecer una propuesta normativa: no deben ser consideradas inferiores o deben serlo. Ese tratamiento tampoco genera conflicto para quien no necesite como criterio de selección de normas la verdad.

El tercer tratamiento, el que habla de una vinculación interna entre los elementos y que por tanto sostiene que son la expresión de concepciones, ¿nos podría llevar a decir que son dos concepciones igualmente valiosas? Pero ese temor es infundado porque ¿desde dónde vamos a concebir ambas como igualmente valiosas o indiferentes? Desde el espacio de significación en el que nos expresamos tenemos también una concepción de lo que es o no igualmente valioso.

Así, el reconocimiento de concepciones diversas, cuando éste es posible, no nos obliga a tratarlas como igualmente valiosas. Como digo, para que esto llegase a producirse el punto de vista de partida (o si se prefiere, la concepción de partida) tendría que permitirlo. Un ejemplo tradicional que permite hablar de expresivismo moral es el de los gustos alimenticios (u otros). La lectura metaética de enunciados morales desde el expresivismo consiente en tratarlos como la manifestación de preferencias. Hay a quien le gusta el gazpacho y hay a quien no le gusta en absoluto. Tan legítima o adecuada es una preferencia como la otra: «sobre gustos no hay nada escrito». No obstante, la crítica más simple a esta postura es aquella que muestra la polémica entre «gustos morales» como un error categorial ${ }^{21}$.

La concepción que tenemos de los gustos alimenticios (o de cualquier otro tipo de gusto) es tal que la pluralidad igualmente aceptable es constitutiva de la noción de gusto y de la práctica sobre gustos. De ahí que no se comparta por todos la concepción que hace de la moral una práctica subjetiva asimilable a la de los gustos personales. Vale decir que la dificultad no es sólo que no se siga del reconocimiento de concepciones diversas sobre lo mismo su equivalencia valorativa, sino que ese reconocimiento, el de que son concepciones sobre lo mismo, es también problemático.

${ }^{21}$ La idea de error categorial en este caso es del tipo de la propuesta por RYLE. G. RYLE, El concepto de lo mental, trad. de E. Rabossi, Buenos Aires, Paidós, 1967, 14. 


\section{UN PAR DE EJEMPLOS}

Es bien conocida la polémica que, en especial en Estados Unidos de América, existe entre los defensores de las teorías evolucionistas, neodarwinistas, y quienes se oponen a ellas esgrimiendo lo que se conoce como explicación del «diseño inteligente». En modo muy breve: quien avala el diseño inteligente sostiene que explicar la presente configuración de la biología humana a partir de las mutaciones azarosas del ADN y el mecanismo de selección natural no resulta algo en modo alguno concluyente. Los miembros del Discovery Institute —radicado en Seattle, http://www.discovery. org- son escépticos respecto de la capacidad explicativa de la teoría de la evolución y creen que merece mayor reconocimiento científico concebir la vida humana como el producto de un diseño inteligente. Si bien insisten en que el diseñador no tiene por qué ser determinada deidad, objeto de culto en una concreta confesión o práctica religiosa, la opción de que un dios haya diseñado el proyecto de la vida, arguyen, no puede excluirse y en sus investigaciones todo apunta precisamente a eso. Se presentan como una concepción científica en biología apartada del Creacionismo. Entre las distintas defensas que esgrimen aparece una específica prueba de la negación elaborada por $\mathrm{P}$. NELSON. El argumento de NELSON viene a ser, también expuesto muy someramente a través de una pregunta, el siguiente: ¿Cómo puede sostenerse que el estatuto epistemológico de la expresión «Todos los organismos que pueblan la Tierra comparten un ancestro común» es científico y simultáneamente que una expresión que no es más que la negación formal de la anterior «No es el caso que todos los organismos que pueblan la Tierra compartan un ancestro común» sea calificado de enunciado religioso?

El ejemplo resulta interesante. Con independencia de la opinión que merezca la polémica y de la postura que se tenga al respecto, lo cierto es que la negación formal de los enunciados generales de este tipo nos enseña algo. Obviamente uno de los enunciados es la negación formal del otro, pero ¿quiere eso decir que también uno es la negación material o sustantiva del otro? Para que así fuese la vinculación entre «todos los organismos que pueblan la Tierra» y «compartir un ancestro común» tendría que ser externa para los participantes de la polémica. Sin embargo, a este nivel de abstracción o generalidad, creo que no puede formularse una oposición teórica, sino de concepciones, esto es, no se están presentando relaciones externas, sino internas. Un neodarwinista no concibe (so pena de no ser un neo-darwinista) a todos los organismos que pueblan la Tierra como organismos que no compartan un ancestro común. Es decir, al hablar de todos los organismos que pueblan la Tierra, habla de seres con un ancestro común; y lo hace porque al usar «organismos que pueblan la Tierra» se compromete con una inferencia material del tipo «si algo es un organismo que puebla la Tierra, entonces comparte un ancestro común con el resto de organismos». Por su parte, quien defiende el diseño inteligente puede separar conceptualmente las nociones involucradas y hacerlo sin mayor dificultad, es más, ése es su propio postulado. Está negando la inferencia material en cuestión.

Resulta ilustrativo ver qué ocurre si a alguien que no participa de la polémica se le pide que «tome partido». Para ello estará interesado, antes de solicitar «pruebas» que avalen las posturas, en entender el carácter del conflicto con un análisis más detenido. Un «observador» que esté intentando comprender la polémica, como yo misma al 
redactar estas líneas, parece que va a tener ciertas posibilidades de éxito en la tarea de convertir las afirmaciones en liza en la expresión de relaciones externas. Puede otorgar un sentido a la expresión «Todos los organismos que pueblan la Tierra» — de ahora en adelante $\mathrm{A}-\mathrm{y}$ un sentido unívoco e independiente para la propiedad relacional «compartir un ancestro común» ${ }^{22}$ - de ahora en adelante B-, y ver la polémica como una en la que un bando dice que $\mathrm{A}$ tiene la propiedad relacional ${ }^{23} \mathrm{~B}$, y otro que ése no es el caso. ¿Qué impide que lo que aquí esté en juego sea la verdad de una afirmación o la de su negación, siendo ambos casos la expresión de una relación externa? En principio nada, sólo que quienes no tienen una concepción determinada al respecto pueden considerar el carácter contingente (teórico o científico si se prefiere) de la vinculación, y eso no es posible para cualquiera. Veamos un último caso.

En la Historia de HeRodoto ${ }^{24}$, para mostrar que las costumbres de los pueblos tienen enorme poder en la conformación de las convicciones más profundas se narra una escena protagonizada por el rey Darío ${ }^{25}$. El rey persa se dirigió a los griegos de su corte preguntándoles por cuánto dinero cambiarían su rito funerario de honra a los padres - consistente en la cremación de los cuerpos- por el rito por el del pueblo dravídico del interior de la India - que era una práctica caníbal puesto que comían sus cuerpos-. Los griegos respondieron que por precio alguno. Después preguntó a los Calatías, el pueblo indio, por cuánto dinero consentirían en quemar a sus progenitores tras su muerte, y éstos también se ofendieron ante lo que entendieron una blasfemia. Ambas comunidades consideraban una aberración la costumbre ajena, y no podían describir aquello que hacían los demás como su forma de honrar a los padres. La descripción física de lo que hacían unos y otros podía coincidir, pero no una en la que se predicase «estar honrando a los padres» ${ }^{26}$. Nótese que una hipotética polémica entre tales indios y griegos puede presentarse como la pugna entre dos concepciones del mismo concepto, o como una disputa verbal acerca de la expresión «honrar a los padres». De hecho, el observador tiene la posibilidad de elegir entre las dos consideraciones, cuando es él quien decide que son «dos formas de hacer lo mismo», o «dos formas distintas de regimentar el uso de "honrar a los padres"». En esta narración Darío ocupa el lugar del observador. No obstante cada pueblo mantiene una concepción que no puede ser «concebida» por el otro. Para los griegos de este pasaje honrar a los padres en un rito funerario está constituido por su cremación. Nada cuenta como rito funerario si no se lleva a cabo esa cremación porque eso ES el rito funerario.

22 Es la idea simple de que mis hermanos y yo compartimos unos progenitores.

${ }_{23}$ Recuérdese que A sustituye a una expresión que presupone una colección de objetos y por ello es posible decir que tiene una propiedad relacional; la tienen los objetos de esa colección.

24 Herodoto, Historia, Libros III y IV, Madrid, Biblioteca Clásica Gredos, 1979, 88-89.

25 «Darius, after he had got the kingdom, called into bis presence certain Greeks who were at hand, and asked: "What he should pay them to eat the bodies of their fathers when they died?". To which they answered, that there was no sum that would tempt them to do such a thing. He then sent for certain Indians, of the race called Callatians, men who eat their fathers, and asked them, while the Greeks stood by, and knew by the belp of an interpreter all that was said: "What he should give them to burn the bodies of their fathers at their decease?". The Indians exclaimed aloud, and bade him forbear such language». HeRODOTUS (440 BCE): The History of Herodotus, trad. de G. Rawlinson, The Internet Classics Archive by Daniel C. Stevenson, http://classics.mit.edu/ Herodotus/history.3.iii.html (consulta realizada el 27 de julio de 2005).

${ }^{26}$ Una afirmación de ese tipo siempre sería un juicio de valor. Vid. R. W. HaLL, «The Controversy of Convention or Nature as the Basis of the State», en Plato, London, George Allen \& Unwin, 1981, 13-30, esp. 17. 
La diferencia entre «existen dos concepciones de lo mismo», o «llaman de la misma forma a dos cosas distintas», sólo tiene sentido cuando hay un modo de identificar lo mismo o lo distinto de manera independiente, y ello nunca puede ser en términos absolutos. No se puede ofrecer un criterio general de qué es igual o distinto (una definición de qué es lo mismo y que es distinto desde ningún lugar). Las distinciones relevantes para ello las ofrece el lenguaje; esto es, un conjunto de conceptos relacionados, tal vez inferencialmente, que adquieren contenido en prácticas de uso.

En una discusión sobre si dos cosas son iguales, ¿cómo se determinaría que estamos en presencia de dos cosas o dos tipos de eventos y no una o uno? Sea lo que sea lo que se haya usado como criterio, será un elemento diferenciador que haga que no pueda decirse que son idénticas. Cuando aquello con lo que hemos determinado - aquello con lo que justificamos- que son dos cosas o fenómenos es un criterio de individuación dentro de una clase, la diferencia existente es irrelevante por lo que hace, obviamente, a la pertenencia a la clase. Si aquello por lo que hemos determinado que son dos objetos es un criterio de identificación, igualarlas supone una clase en la que incluir ambas subclases. Así las cosas, podemos decir que estamos ante propuestas o concepciones distintas, pero ¿cómo se elige entre concepciones? Hay quien diría que todo depende del significado de nuestras palabras y que éste a su vez tiene carácter meramente estipulativo. Bastaría pues con estipular el sentido de nuestras expresiones. Yo creo que elegir o secundar una concepción se parece más a cómo nos relacionamos con las personas. Con la mayoría de personas no nos relacionamos porque no las hemos conocido ni las conoceremos. Con algunas personas que hemos conocido superficialmente no nos queremos relacionar y con otras nos atrae la idea de hacerlo por lo que hemos creído entrever. De algunas otras, que conocemos bien, podemos pensar que más valdría no haberlas conocido. Y, con suerte, siempre hay alguien que nos intriga, seduce e inspira más confianza y entusiasmo cuanto más lo conocemos. Creo que elegir entre concepciones es algo mucho más complejo que elegir entre definiciones estipulativas. 